\title{
Separate-component-stabilization system for protein and DNA production without the use of antibiotics
}

\author{
Cédric Y. Szpirer and Michel C. Milinkovitch
}

BioTechniques 38:775-781 (May 2005)

\begin{abstract}
Plasmid instability is a significant concern in the industrial utilization of microorganisms for protein or DNA production. Here we report on the development of a new and highly effective stabilization system based on the use of the ccd antidote/poison genes. For the first time, we separated the antidote gene from the poison gene: localizing the former in the plasmid and integrating the latter in the bacterial chromosome. We show that this separate-component-stabilization (SCS) strategy: (i) allows for perfect stabilization without the use of antibiotics; (ii) increases three to five times the recombinant protein production levels; and (iii) does not require any specific modification of the protein production process or culture medium. We illustrate that point by using the classical T7 promotor (i.e., used in most expression systems). Finally, we demonstrate that the SCS system increases by five the yield in DNA production, a result especially important for the design and production of gene therapy constructs void of any antibiotic resistance gene.
\end{abstract}

\section{INTRODUCTION}

Plasmid instability is a significant concern in the academic and industrial utilization of microorganisms for protein or DNA production. Usually, these processes require the use of a bacterial plasmid construct as a vector carrying a gene to be expressed. It has been demonstrated that the growth rate of plasmid-bearing cells is significantly reduced relative to that of a plasmidfree cells, simply because plasmid replication and transcription, as well as protein production, represent a significant burden on cellular metabolism (1-4). Hence, in a fermentation process, cells losing the plasmidic construct exhibit a higher fitness than construct-free cells, and the former rapidly overcome the latter in the bacterial population. Antibiotic resistance genes are the most commonly used selectable markers in fermentation procedures to avoid plasmid free cells to survive and dominate the culture. However, antibiotics are expensive compounds, and they (or their degradation products) can contaminate the biomass or production product. Given that these contaminations are unacceptable from industrial and medical or regulatory perspectives [see Food and Drug Administration (FDA) recommendations; Docket nos. 94D0259 and 98D-0374], the assessment of the residual antibiotics levels and, if necessary, their subsequent removal are costly procedures. Moreover, the use of antibiotics in intensive culture conditions (high biomass and/or continuous culture) is not efficient due to dilution or antibiotic inactivation $(2,5)$.

Several alternative strategies have been developed to reduce the risk that plasmid-free cells overtake a culture. One approach is the complementation of an essential mutated chromosomal gene by a wild-type allele inserted into a plasmid. For example, Wang and coworkers (6) developed such a system in which the mutant host is unable to synthesize an essential amino acid without a plasmid carrying the gene that provides this function. However, this approach seriously restricts the possible choices in growth medium and, for example, prevents the use of rich media. Recently, a strategy was developed $(7,8)$ in which a plasmidmediated repressor titration overcomes the repression of an essential chromosomal gene placed under the control of the lac operator. However, this procedure has the following serious limitations: (i) it makes the Lac promoter (and derivatives) unavailable for other purposes such as protein expression; (ii) the system is limited to Escherichia coli or other bacteria in which the Lac promoter is functional; and (iii) medium containing lactose must be avoided.

Yet another strategy is to use antidote/poison genes (i.e., postsegregational killing genes) that induce host killing upon plasmid loss (9-11). These systems are naturally found on plasmids, chromosomes, and bacteriophages. An antidote/poison system is typically organized as an operon and composed of two genes: one encoding a stable poison protein and the other encoding an unstable antidote protein. In some systems, the antidote is an antisense RNA that controls the expression of the poison 
Table 1. Sequences and Names of Primers Used for Building Chromosomal and Plasmidic Constructs

\begin{tabular}{|ll|}
\hline Primer & Sequence \\
\hline PmobccdA & 5'-GGGAGGGGCAGGCATGAAGCAGCGTATTACAGTG-3' \\
ccdAendSphl & 5'-CCGCATGCTCACCAGTCCCTGTTCTCG-3' \\
CS4Sphl & $5^{\prime}$-GAGCATGCGTTGTCCACGTTGTCCACGGGCCGAGCG-3' \\
ccdApMob & $5^{\prime}$-GTAATACGCTGCTTCATGCCTGCCCCTCCCTTTTGG-3' \\
ccdcat & $5^{\prime}$-CCTTAAACTGCATAGCACACCTCTTTTTGAC-3' \\
cat1 & $5^{\prime}$-CGAATAAATACCTGTGACGG-3' \\
ccdBend & $5^{\prime}$-TTGGGAATTCCTCCTGCTAGCCTGACATTTATATTCCC-3' \\
Catccd & $5^{\prime}$-GAGGTGTGCTATGCAGTTTAAGTTTACAC-3' \\
Rev & $5^{\prime}$-CTTATCGTGAACGTCGGCCATGTTGTGCCTCTTGCTGACGCAACGCCACCAGGAAACAGCTATGACCATG-3' \\
Fwd & $5^{\prime}$-CTATGCAGGAAAATATATCAGTAACCGATTCATACAGCACCGGGAATGCCTTGTAAAACGACGGCCAGTG-3' \\
The italicized sequences in the Rev and Fwd primers are the sequences used for the targeting of the homologous recombination into the dcm gene.
\end{tabular}

gene (hok/sok from the R1 plasmid, pndAB from $\mathrm{R} 483$ ). In previously developed stabilization systems using antidote/poison genes, the sequence encoding the full operon is cloned into the plasmid to be stabilized $(10,11)$. In plasmid-cured bacteria, de novo synthesis of the antidote/poison pair ends, and as a result of antidote degradation, the poison is free to exert its toxicity, eventually causing cell death. The F-plasmid-derived $c c d$ operon is one of such systems. The $\mathrm{CcdB}$ poison gene has already been used in DNA cloning technology for the selection of recombinants (12-14) and is now used as an essential component in several molecular cloning tools and kits such as the Gateway ${ }^{\circledR}$ Technology (Invitrogen, Carlsbad, CA, USA). Unfortunately, these antidote/poison systems can only delay, but not prevent, the takeover of a culture by plasmid-free cells (10). Indeed, after plasmid loss, dilution of the poison by bacterial growth before complete antidote degradation can allow cells to survive and produce a high-fitness line of plasmid-free cells.

Here we describe and validate a new and highly effective stabilization system based on the use of the $c c d$ antidote/ poison genes. For the first time, we separated the antidote gene from the poison gene-localizing the former in the plasmid and integrating the latter in the bacterial chromosome. We show that this separated-component-stabilization (SCS) strategy: ( $i$ ) allows for perfect stabilization without the use of antibiotics during the production process; (ii) increases three to five times the recombinant protein production levels; and (iii) does not require any specific modification of the DNA or protein production process or culture medium.

\section{MATERIALS AND METHODS}

\section{Bacteria and Plasmids}

The pULB2710 plasmid was constructed by cloning the complete $c c d A B$ operon into pMLO59 (15), a vector that bears the spectinomycin resistance gene. The BL21(DE3) bacterial strain (16), containing the T7 RNA polymerase gene integrated into its chromosome, was purchased from Novagen (Merck KGaA, Darmstadt, Germany). The DH10B strain was purchased from Invitrogen. The pET21a+ plasmid (Novagen) carries the ampicillin resistance gene.

\section{PCR Amplification, Cloning, and Insertion}

The sequences of all PCR primers are listed in Table 1. The $c c d A$ gene and the mob promoter were amplified by PCR using, respectively, the primer pairs PmobccdA plus ccdAendSphI and CS4SphI plus ccdApMob. The products were fused by PCR overlap extension using the primers CS4SphI and ccdAendSphI, each containing the $S p h I$ restriction site. The resulting PCR product was cleaved by $S p h \mathrm{I}$ and cloned into both the pMLO59 plasmid (17) and the pET21a+ vector (Novagen), yielding the pMLOCcdA and pETstabil1 constructs, respectively. The pETTraG plasmid (18), harboring the traG gene, was restricted by HindIII and BamHI. The fragment corresponding to the $\operatorname{tra} G$ gene was cloned into the pETstabil1 vector digested by the same enzymes resulting into the pETstabilTraG plasmid (the $\operatorname{tra} G$ gene is fused, in $3^{\prime}$, to six histidine codons). Using the pDONR207 vector (Invitrogen) as a template, the chloramphenicol-acetyltransferase (cat) gene and the promoter from the $c c d$ operon were co-amplified with the ccdcat and cat1 primers, while the $c c d B$ gene was amplified with the primers ccdBend and catccd (Table 1). The two fragments were fused using PCR overlap extension with the primers cat1 and ccdBend (Table 1), and the resulting amplification product was cloned into the pCRXL-TOPO vector (Invitrogen), yielding the pCatccdB construct. The $c a t-c c d B$ fragment was next amplified by PCR from pCatccdB using the primers Rev and Fwd (Table 1) and introduced by electroporation and homologous recombination into the $d \mathrm{~cm}$ gene of the NM519 strain (19) containing pETstabil1 (the first 50 bases of the primers are identical to the $5^{\prime}$ and $3^{\prime}$ ends of the $d c m$ target gene). The fragment inserted was next transduced using P1vir bacteriophage (for a detailed protocol see Reference 20) into the DH10B strain containing the pMLOCcdA plasmid and into BL21(DE3) containing the pETstabilTraG plasmid. The recombinants were selected using the chloramphenicol resistance. The DH10B and BL21(DE3) strains, each containing the $c c d B$ gene in their chromosome, were called CYS21 and SE1, respectively. 
Table 2. Stability of Thermosensitive Plasmids After 20 Generations of Growth Without Antibiotics

\begin{tabular}{|llccc|}
\hline Strain & Plasmid & \multicolumn{2}{c}{$\begin{array}{c}\text { Population } \\
\text { (bacteria/mL) }\end{array}$} & $\begin{array}{c}\text { Stability } \\
\text { (\%) }\end{array}$ \\
\cline { 3 - 4 } & & Total & With Plasmid & \\
\hline DH10B & pMLO59 & $3.3 \times 10^{9} \pm 2 \times 10^{8}$ & $1.4 \times 10^{7} \pm 1 \times 10^{7}$ & 0.42 \\
DH10B & pULB2710 & $2.4 \times 10^{9} \pm 2 \times 10^{8}$ & $6.4 \times 10^{8} \pm 3 \times 10^{8}$ & 26.50 \\
CYS21 & pMLOCcdA & $8.6 \times 10^{8} \pm 5 \times 10^{8}$ & $8.6 \times 10^{8} \pm 5 \times 10^{8}$ & 100 \\
After 20 generations, bacterial cultures were plated on nonselective and selective media to quantify, \\
respectively, the total population and the subpopulation of plasmid-bearing cells. The pMLO59 plasmid \\
is free of any stabilization system. The pULB2710 plasmid encodes the ccd operon (ccdA and $c c d B$ ). \\
$\begin{array}{l}\text { The pMLOccdA plasmid (including the antidote ccdA gene) was used with the CYS21 strain, a DH10B } \\
\text { strain that contains the ccdB poison gene in its chromosome. The experiment was repeated three times, } \\
\text { standard deviations are indicated. }\end{array}$ \\
\hline
\end{tabular}

\section{Stability Test}

Single colonies were grown overnight in $10 \mathrm{~mL} \mathrm{LB}$ medium without antibiotic at $37^{\circ} \mathrm{C}$. The following day, $1 \mu \mathrm{L}$ from each culture was used for inoculation of $10 \mathrm{~mL}$ fresh LB medium. Cultures were grown overnight at $37^{\circ} \mathrm{C}$. The following day, bacterial cultures were diluted, and each dilution was plated on nonselective (LB) and selective (LB medium plus spectinomycin $100 \mu \mathrm{g} / \mathrm{mL}$ or ampicillin 100 $\mu \mathrm{g} / \mathrm{mL}$ ) medium in order to estimate the total population and the population containing the plasmid.

\section{Protein Production}

Small-scale expression. One hundred microliters of an overnight culture $\left(5 \times 10^{8}\right.$ bacteria) of the BL21(DE3) strain carrying the pETTraGhis plasmid (18) were inoculated into $10 \mathrm{~mL} \mathrm{LB}$ medium supplemented with ampicillin (100 $\mu \mathrm{g} / \mathrm{mL}$ ). Similarly, $100 \mu \mathrm{L}$ of an overnight culture $\left(5 \times 10^{8}\right.$ bacteria $)$ of BL21(DE3) containing both the $c c d B$ gene in its chromosme (SE1 strain) and the pETstabilTraG plasmid were inoculated into $10 \mathrm{~mL}$ LB medium without antibiotic. Both cultures were grown at $37^{\circ} \mathrm{C}$. When an optical density $(600 \mathrm{~nm})$ of 0.8 was reached (8 $\times 10^{8}$ bacteria $/ \mathrm{mL}$, see Table 3 ), protein production was induced using $1 \mathrm{mM}$ isopropyl- $\beta$-D-thiogalactopyranoside (IPTG). After $2 \mathrm{~h}$ of incubation, protein extracts were performed as follows: 50 $\mu \mathrm{L} 100 \%$ trichloroacetic acid (TCA) solution were added to $1 \mathrm{~mL}$ culture, and cells were pelleted through centrifugation and washed twice with cold acetone. The pellet was resuspended in sodium dodecyl sulfate (SDS) gel electrophoresis sample buffer. The gel was scanned, and the raw signal corresponding to each band was measured using the ImageQuant ${ }^{\mathrm{TM}}$ TL software (Amersham Biosciences, Piscataway, NJ, USA). The intensity of each band was normalized against a $40-\mathrm{kDa}$ band that is present in all lanes and whose intensity is independent of induction. The proportion of the protein of interest was also compared to the total amount of protein in each lane rather than to the single 40-kDa band.

Protein and DNA productions after 30 generations. One microliter from each overnight culture was used to inoculate $10 \mathrm{~mL} \mathrm{LB}$ medium, the cultures were grown overnight at $37^{\circ} \mathrm{C}$, and this procedure was iterated twice. Then, $100 \mu \mathrm{L}$ of each of the last (third) overnight cultures were used for protein induction as described above.

\section{RESULTS}

\section{Strategy}

We separated the $c c d A$ antidote gene from the $c c d B$ poison gene in order to obtain complete stabilization of plasmids in bacteria (Figure 1). On one hand, we introduced the $c c d A$ antidote gene into the plasmid under the control of the mob promoter isolated from pBBR1. The mob gene codes for a mobilization protein (Mob) and is autoregulated by this protein; thus in the absence of the Mob protein, the mob promoter is continuously but moderately active (21). In our system, the Mob protein being absent, $c c d A$ is constitutively expressed. On the other hand, the $c c d B$ poison gene was fused downstream to the $c c d$ promoter, and this construction was introduced into the bacterial chromosome. In the presence of antidote, the $c c d$ promoter is strongly repressed (by the antidote/ poison complex; see Reference 22), hence, the resulting very low amount of poison greatly reduces the likelihood of emergence of cells harboring a mutated $c c d B$ gene coding for an inactive $\mathrm{CcdB}$ protein. If the plasmid is lost, the antidote is degraded (by the Lon protease; see Reference 15), and the production of the toxin is activated, causing cell death; the $c c d B$ poison gene blocks the E. coli DNA gyrase (23).

\section{Stability Tests}

To better evaluate the efficiency of our SCS system, we used the pMLO59 thermosensitive vector, which is particularly prone to be lost during cell division because it is not, or poorly, replicated above $30^{\circ} \mathrm{C}(17)$.

The stability of the pMLOccdA plasmid (containing the $c c d A$ gene under the control of the Pmob promoter) was compared to those of: (i) the same vector containing the complete $c c d$ operon (pULB2710) and (ii) the original vector (pMLO59). The pMLOccdA plasmid was tested using the CYS21 strain (DH10B containing the $c c d B$ poison gene in the chromosome), while the pULB2710 and pMLO59 plasmids were tested in the wild-type DH10B strain. After 20 generations, bacterial cultures were diluted and plated on nonselective and selective media to quantify the respective sizes of the total population and of the subpopulation of plasmidbearing cells. Table 2 indicates that: $(i)$ the total population size is maximum for the control culture (pMLO59: $3 \times$ $10^{9}$ bacteria $/ \mathrm{mL}$ ) and (ii) the population size is somewhat reduced when a stabilization system based on a plasmid containing the $c c d$ operon (pULB2710: $2 \times 10^{9}$ bacteria $/ \mathrm{mL}$ ) or the $c c d A$ gene (pMLOccdA: $9 \times 10^{8}$ bacteria $\left./ \mathrm{mL}\right)$ is used. This result is easily explained by the fact that plasmid-free cells are killed, thereby decreasing the total number of bacteria. However, the important parameter in a DNA or protein production process is certainly 
Table 3. Effect of Growth (30 Generations) and Expression (During 2 h) on Plasmid Stability in the Absence of Antibiotics

\begin{tabular}{|lcccc|}
\hline Strain and Plasmid & $\begin{array}{c}\text { Population Before Induction } \\
\text { (bacteria/mL) }\end{array}$ & $\begin{array}{c}\text { Population After Induction } \\
\text { (bacteria/mL) }\end{array}$ \\
\cline { 2 - 5 } & Total & With Plasmid & Total & With Plasmid \\
\hline BL21DE3(pETTraG) & $7.0 \times 10^{8} \pm 7 \times 10^{7}$ & $2.2 \times 10^{8} \pm 4 \times 10^{7}$ & $1.0 \times 10^{9} \pm 2 \times 10^{8}$ & $1.4 \times 10^{6} \pm 5 \times 10^{5}$ \\
SE1 (pETstabilTraG) & $8.0 \times 10^{8} \pm 5 \times 10^{7}$ & $8.0 \times 10^{8} \pm 4 \times 10^{7}$ & $2.2 \times 10^{8} \pm 3 \times 10^{7}$ & $2.1 \times 10^{8} \pm 3 \times 10^{7}$ \\
After 30 generations, bacterial cultures were plated on nonselective and selective media to quantify, respectively, the total \\
population of bacteria and the subpopulation of plasmid-bearing bacteria before and after an induction period of 2 h. The \\
experiment was repeated three times, and the standard deviation was calculated. SE1 is a BL21(DE3) strain that includes \\
the ccdB poison gene in its chromosome. pETstabilTraG is a pETTraG plasmid that includes the ccdA antidote gene.
\end{tabular}

not the total bacterial population size but the number of cells harboring the plasmid construct of interest. In that respect, both stabilization systems are efficient: the population of plasmidbearing cells is $8.6 \times 10^{8}$ bacteria $/ \mathrm{mL}$ for pMLOccdA and $6.4 \times 10^{8}$ bacteria/ $\mathrm{mL}$ for pULB2710 (i.e., respectively, 61 and 46 times more than the $1.4 \times 10^{7}$ bacteria/mL for the pMLO59 control). However, although the pULB2710 system (plasmid with the full $c c d$ operon) is efficient at increasing the proportion of plasmid-bearing cells, $73.5 \%$ of the cells still escape the system and have lost the construct of interest. Hence, given the selective advantage of fast-growing plasmid-free cells, further growth of such a colony (i.e., for more than the 20 generations for which the test was made) can only increase the proportion of plasmid-free cells (see below for additional results). In that respect, the SCS system reported here (pMLOccdA) is remarkably efficient, as we could not detect any cell lacking the ccdA-containing plasmid (Table 2). Hence, our SCS system provides perfect stabilization-separation of the antidote and the poison genes in the plasmid and chromosome, respectively, is significantly more efficient than the use of a complete antidote/poison operon in the plasmid.

\section{Plasmid Stabilization and T7 Expression System}

We applied the SCS system to protein production in the BL21(DE3) strain (16) containing a vector (pET21) designed for protein expression using the T7 RNA polymerase. The cassette encoding the $c c d A$ gene under the control of the Pmob promoter was cloned into pET21 to yield a vector that we called pETstabill (see Materials and Methods section). To investigate the impact of our system on protein production, we cloned the gene encoding the $69-\mathrm{kDa}$ TraG bacterial protein tagged by 6 histidines in both pETstabill and the nonmodified vector pET21 (encoding the ampicillin resistance gene), resulting in the pETstabilTraG and pETTraG constructions, respectively. The TraG protein is involved in conjugation and mobilization processes and is known as nontoxic (18). The overproduction of TraG using the $\mathrm{pET}$ stabilTraG construct in a BL21(DE3) strain containing the $c c d B$ gene inserted into its chromosome and without the use of antibiotics was compared to the overproduction of TraG using the pETTraG construct in a nonmodified BL21(DE3) strain in the presence of ampicillin (small-scale expression). After protein gel electrophoresis, the amounts of $\mathrm{TraG}$ protein that were produced in the two conditions were quantified and compared (see Material and Methods section). Figure 2A indicates that, after $2 \mathrm{~h}$ of induction, significantly more TraG protein (and a lower background) is produced using our SCS system (Figure 2A, lane 3) compared to the antibiotic-dependent classical system (Figure 2A, lane 2). Moreover, it is important to note that no overproduction of the antidote is detectable, such that a negligible amount of amino acids is immobilized for the functioning of the SCS system.

Because stabilization systems without antibiotics are of particularly high interest for large-scale fermentations, we tested plasmid stability and protein production after 30 generations without antibiotics. As shown in Figure $2 \mathrm{~B}$, protein production is three to five times higher with our SCS system (Figure 2B, lane 3) than using the conventional strain (Figure 2B, lane 4). This result indicates that the pET21 plasmid tested here is unstable despite that: $(i)$ it is a medium-copy number plasmid (it bears a pBR322 replication gene) and (ii) it encodes a nontoxic protein. Table 3 illustrates that the SCS system is fully insensitive to instability induced by overexpression; $2 \mathrm{~h}$ after induction of protein production, no cell has escaped the SCS system (i.e., with antidote and poison genes as separate genetic elements) as $100 \%$ of the cells exhibit the plasmidic construct. On the contrary, the conventional strain (without poison in the chromosome)

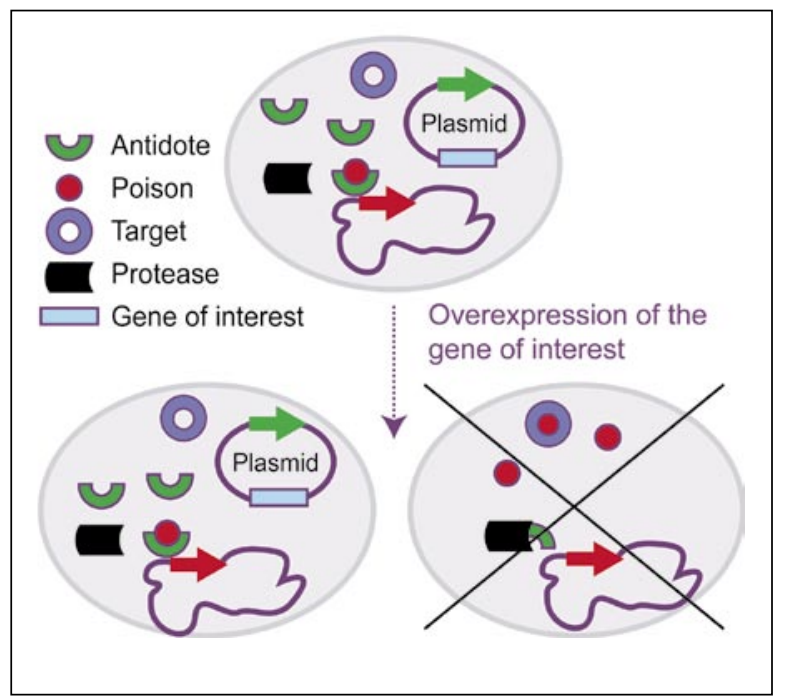

Figure 1. Principle of the separated-component-stabilization (SCS) system. The antidote gene $(c c d A)$ is introduced into the plasmid under the control of a constitutive promoter. On the other hand, the poison gene $(c c d B)$ is introduced into the chromosome of the bacteria. Expression of the poison gene is under the control of a promoter strongly repressed in the presence of the plasmid. When the plasmid is lost, the antidote is degraded (or diluted during cell division), and the production of the toxin is induced, causing cell death. 
experiences a population shift from an already moderate $(31 \%)$ to a very low $(0.14 \%)$ proportion of plasmid-bearing bacteria.

The new stabilization system reported here (SCS) is also likely to be highly effective for the production (amplification) of cloned genes. Indeed, since the plasmid is unstable even in the absence of induction of protein expression (see Table 3 ), it is reasonable to assume that the output of DNA production will also be increased using our stabilization system. To test this hypothesis, cultures were grown for 30 generations, and plasmid DNA was extracted and quantified. As shown in Figure 3, the DNA yield is five times higher with (Figure 3, lanes 2 and 4) than without (Figure 3, lanes 1 and 3) our stabilization system. This result is especially important for the preparation of plasmids as vectors in gene therapy. Indeed, the use of plasmids bearing an antibiotic resistance gene is particularly unwanted in this case.

\section{DISCUSSION}

We have developed a new stabilization system based on the separation of a poison gene and its corresponding anti-poison gene. These systems have already been used for the selection of recombinants in cloning processes (1214). The major advantages of antidote/ poison systems are: $(i)$ their small size (about 100 amino acids for the poison and 90 amino acids for the antidote); (ii) the efficiency of the poison selected to exert its activity in bacteria (resistantmutant are rare or nonexistent); and (iii) their broad range of use; some antidote/poison systems are active in several Gram-negative bacteria (24) or even in yeast and mammalian cells [ relBE $(25,26)$ and kis-kid (27)]. Although systems using these genes $(10,12)$ or based on other strategies (68 ) have been demonstrated to increase plasmid stability, they do not provide fully efficient solutions because they either cannot prevent the takeover of a culture by plasmid-free cells (10) or they require specific culture media. The SCS system described here yields perfect plasmid stabilization during DNA or protein productions and is functional in any culture medium and any production process. We demonstrate, using stability tests, that the separation of the antidote and poison genes is more efficient than the use of a complete antidote/poison operon; the SCS system increases the absolute number of plasmid-bearing cells by two orders of magnitude (in comparison to a control without any stabilization system) after $2 \mathrm{~h}$ of a nontoxic bacterial protein overproduction. In consequence, our SCS system allows, without the use of any antibiotic during the production process, a 3- to 5-fold increase in the amount of DNA or protein produced.

It is remarkable that, by comparison with a conventional system, the use of the SCS system allows not only the killing of the cells that have lost the plasmid, but also increases the number of plasmid-bearing cells. This 
can easily be explained by the fact that inhibiting the growth of plasmid-free cells makes all resources available to plasmid-bearing bacteria. Moreover, cell lysis of the plasmid-free cells could even stimulate growth of surviving (plasmid-bearing) cells, as previously shown by Corchero and coworkers (28).

We adapted our SCS system to the T7 expression system developed by Studier and coworkers (16). The $\mathrm{T} 7$ system requires a bacterial strain

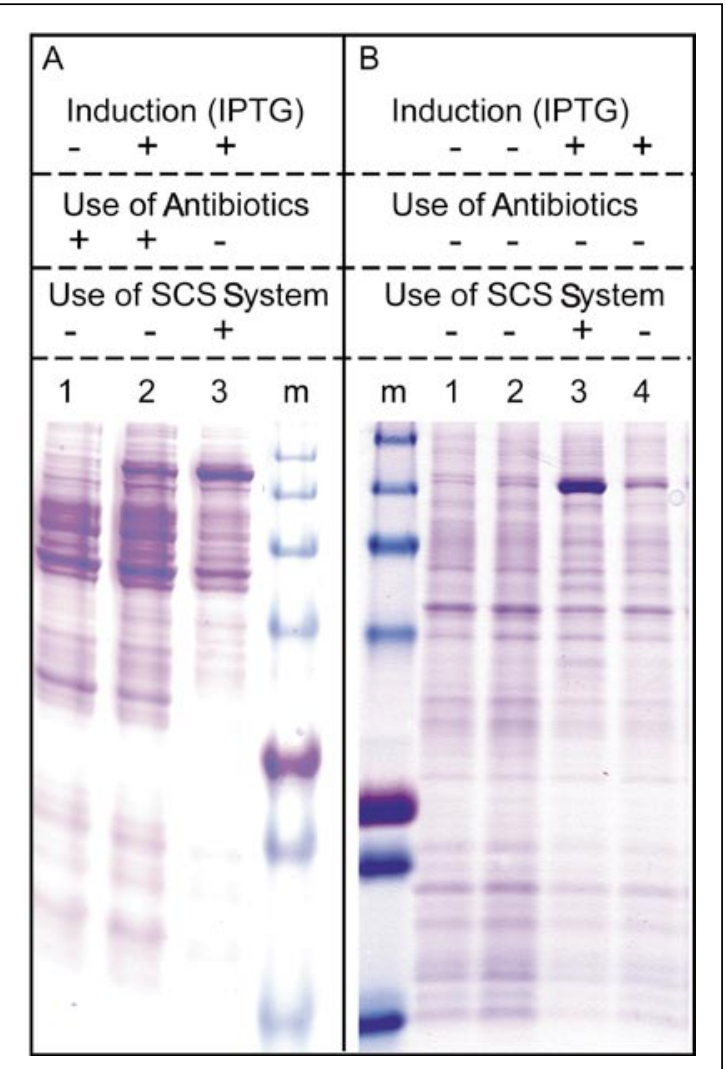

Figure 2. Protein expression using the separated-componentstabilization (SCS) system compared to a conventional system. (A) Overexpression of the $69-\mathrm{kDa}$ TraGhis protein after 10 generations. Electrophoresis of the total protein extracts in a sodium dodecyl sulfate (SDS) $16 \%$ polyacrylamide gel. Lane 1, non-induced BL21(DE3) (containing the pETTraG plasmid) in presence of antibiotics; lane 2, induced BL21(DE3) containing the pETTraG plasmid in presence of antibiotics; lane 3, induced SE1 [i.e., a BL21(DE3) strain containing the $c c d B$ gene in its chromosome] containing the pETStabilTraG plasmid in absence of antibiotics; lane $\mathrm{m}$, SeeBlue ${ }^{\circledR}$ Plus 2 protein size standards in $\mathrm{kDa}: 98,64$, $50,36,22,16$, and 6 (Invitrogen). (B) Overexpression of the 69$\mathrm{kDa}$ TraGhis protein in the absence of antibiotics after 30 generations. Electrophoresis of the total protein extracts in an SDS 16\% polyacrylamide gel. Lane $\mathrm{m}$, protein size standards (as in panel A); lane 1, non-induced SE1 containing pETstabilTraG; lane 2, non-induced BL21(DE3) containing pETTraG; lane 3, SE1 containing pETstabilTraG after $2 \mathrm{~h}$ of induction; lane 4, BL21(DE3) containing pETTraG after $2 \mathrm{~h}$ of induction. IPTG, isopropyl- $\beta$-Dthiogalactopyranoside.
E. coli RelE protein from the relBE system that has been shown to be toxic in mammalian and yeast cells (when associated to, respectively, a mammalian or yeast promoter; see References 25 and 26). Furthermore, it is crucial to realize that: $(i)$ the poison gene is induced only in cells that lose the plasmid, and (ii) these cells will die (i.e., they will not generate new plasmid-free cells expressing the poison). Hence, only minute amounts of the poison protein are released in the supernatant, which is then discarded during downstream processing. Furthermore, the poison and/or antidote proteins $(\mathrm{CcdB}$ and $\mathrm{CcdA}$ in the SCS system) are very small (8.7 and 11.7 $\mathrm{kDa}$, respectively) and can therefore be easily removed from the protein extract during downstream purification (most proteins of interest have much higher molecular weights).

In conclusion, the new system reported here, based on the separation of the poison and antidote genes (hence the term SCS), appears as an excellent tool for increasing the yield of protein or DNA production without the use of

$(\mathrm{CcdB})$, one might that products extracted from the bacterial culture could be contaminated by the poison protein and, as such, acquire some toxicity. However, E. coli strains currently used in bacterial fermentation processes all contain several antidote/ poison operons in their chrosomose and plasmids. For example, the poison and antidote genes used here for the design of the SCS system are present in several E. coli strains commonly used for protein or DNA production (the $c c d A B$ operon is present in the $\mathrm{F}$ plasmid), and to our knowledge, it has never been reported that the presence of the $\mathrm{F}$ plasmid is toxic. This result is not surprising as the $\mathrm{CcdB}$ protein does not seem to be toxic in eukaryote cells (inactive on mammalian topoisomerase II; see Reference 30), unlike the

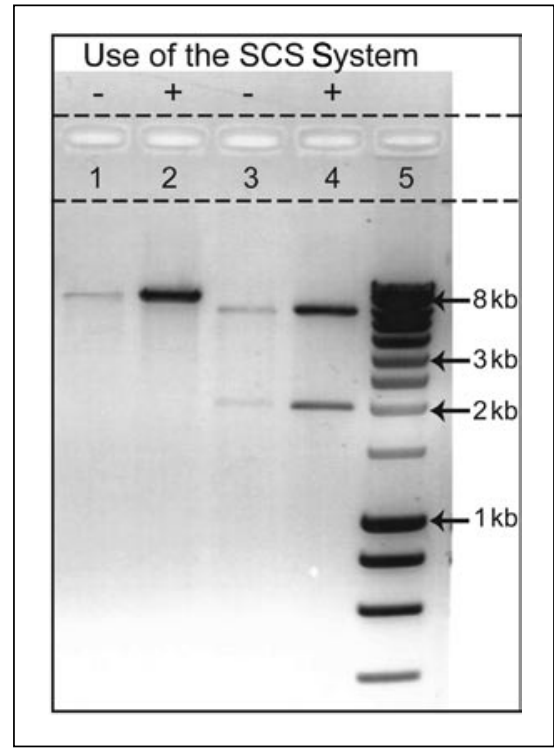

Figure 3. Plasmid DNA extractions cultures (after 30 generations) with and without the separated-component-stabilization (SCS) system. Electrophoresis of plasmid DNA in a $1 \%$ agarose gel. Lanes 1 and 2, BamHI restriction (unique site) of the pETTraG and pETstabilTraG plasmids extracted from BL21(DE3) and SE1, respectively; lanes 3 and 4, StyI restriction (two sites) of the plasmids extracted from BL21(DE3) and SE1, respectively; lane 5, DNA molecular size marker. 
antibiotics. Moreover, the system can be combined with basically any proteinexpression or DNA production method without modification of the process or culture medium (as illustrated here with the T7 expression system). The exact productivity gain provided by the SCS system will, of course, depend on the specific production system and overexpressed protein. However, a system providing perfect stabilization, as does our SCS system, will also increase reproducibility, and thus standardization, of the process.

Finally, it has been recently shown that poison and antidote genes exist in all bacterial genomes sequenced to date and that some of them are active in eukaryote cells. The stabilization system described here should thus be adaptable to other bacterial strains and species (by using appropriate poison and antidote genes) and even in yeast or mammalian cells. We are currently adapting the SCS system to these production processes.

\section{ACKNOWLEDGMENTS}

We thank Drs. L. Van Melderen and M. Faelen for providing the $p U L B 2710$ and pMLO59 plasmids. We thank Dr. P. Gabant for critical reading and interesting discussions. This work was supported by the Region Wallonne (BioVal, Biorobot-Initiative 114840) and the National Fund for Scientific Research (FNRS), Belgium. C.Y.S. is a Postdoctoral Researcher of the FNRS.

\section{COMPETING INTERESTS STATEMENT}

The authors declare no competing interests.

\section{REFERENCES}

1.Betenbaugh, M., C. Beaty, and P. Dhurjati. 1989. Effects of plasmid amplification and recombinant gene expression on the growth kinetics of recombinant $E$. coli. Biotechnol. Bioeng. 33:1425-1436.

2.Baneyx, F. 1999. Recombinant protein expression in Escherichia coli. Curr. Opin. Biotechnol. 10:411-421.

3.Corchero, J.L. and A. Villaverde. 1998. Plasmid maintenance in Escherichia coli recom- binant cultures is dramatically, steadily, and specifically influenced by features of encoded proteins. Biotechnol. Bioeng. 58:625-632.

4.Glick, B. and J. Pasternak. 2003. Molecular Biotechnology: Principles and Applications of Recombinant DNA, 3rd ed. ASM Press, Washington.

5.Mari, Y.M., A.E. Espinosa, R. Ubieta, O.F. Batista, and M.L. Fernandez. 1999. Effect of the selection marker on the viability and plasmid stability of two human proteins with neurotrophic action expressed in Escherichia coli. Biochem. Biophys. Res. Commun. 258:29-31.

6.Wang, M.D., L. Buckley, and C.M. Berg. 1987. Cloning of genes that suppress an Escherichia coli $\mathrm{K}-12$ alanine auxotroph when present in multicopy plasmids. J. Bacteriol. 169:5610-5614.

7.Williams, S.G., R. Cranenburgh, A.M. Weiss, C.J. Wrighton, D.J. Sherratt, and J.A. Hanak. 1998. Repressor titration: a novel system for selection and stable maintenance of recombinant plasmids. Nucleic Acids Res. 26:2120-2124

8.Cranenburgh, R., J. Hanak, S. Williams, and D. Sheratt. 2001. Escherichia coli strains that allow antibiotic-free plasmid selection and maintenance by repressor titration. $\mathrm{Nu}$ cleic Acids Res. 29:26-32.

9.Wood, T.K., R.H. Kuhn, and S.W. Peretti. 1990. Enhanced plasmid stability through post-segregational killing of plasmid-free cells. Biotechnology Techniques 4:39-44.

10.Pecota, D., C. Kim, K. Wu, K. Gerdes, and T. Wood. 1997. Combining the hok/sok, parDE, and pnd postsegregational killer loci to enhance plasmid stability. Appl. Environ. Microbiol. 63:1917-1924.

11.Cameron, B. and J. Crouzet. 1995. Method for the production of recombinant proteins, plasmids and modified cells. Patent no. WO96/08572. Rhone-Poulenc Rorer SA, France.

12.Gabant, P., P.L. Dreze, T. Van Reeth, J. Szpirer, and C. Szpirer. 1997. Bifunctional lac $\mathrm{Z}$ alpha- $c c d B$ genes for selective cloning of PCR products. BioTechniques 23:938-941.

13.Gabant, P., C.Y. Szpirer, M. Couturier, and M. Faelen. 1998. Direct selection cloning vectors adapted to the genetic analysis of gram-negative bacteria and their plasmids. Gene 207:87-92.

14.Bernard, P., P. Gabant, E.M. Bahassi, and M. Couturier. 1994. Positive-selection vectors using the F plasmid $c c d B$ killer gene. Gene 148:71-74.

15.Van Melderen, L., P. Bernard, and M. Couturier. 1994. Lon-dependent proteolysis of CcdA is the key control for activation of $\mathrm{CcdB}$ in plasmid-free segregant bacteria. Mol. Microbiol. 11:1151-1157.

16.Studier, F., A. Rosenberg, J. Dunn, and J. Dubendorff. 1990. Use of T7 RNA polymerase to direct expression of cloned genes. Methods Enzymol. 185:60-89.

17.Clerget, M. 1991. Site-specific recombination promoted by a short DNA segment of plasmid R1 and by a homologous segment in the terminus region of the Escherichia coli chromosome. New Biol. 3:780-788.

18.Szpirer, C.Y., M. Faelen, and M. Couturier.
2000. Interaction between the RP4 coupling protein TraG and the pBHR1 mobilization protein Mob. Mol. Microbiol. 37:1283-1292.

19.Sain, B. and N.E. Murray. 1980. The hsd (host specificity) genes of E. coli K 12. Mol. Gen. Genet. 180:35-46.

20.Miller, J. 1972. Experiments in Molecular Genetics. CSH Laboratory Press, Cold Spring Harbor, NY.

21.Szpirer, C.Y., M. Faelen, and M. Couturier. 2001. Mobilization function of the pBHR1 plasmid, a derivative of the broad-host-range plasmid pBBR1. J. Bacteriol. 183:2101-2110.

22.Salmon, M.A., L. Van Melderen, P. Bernard, and M. Couturier. 1994. The antidote and autoregulatory functions of the F plasmid CcdA protein: a genetic and biochemical survey. Mol. Gen. Genet. 244:530-538.

23.Bernard, P. and M. Couturier. 1992. Cell killing by the F plasmid CcdB protein involves poisoning of DNA-topoisomerase II complexes. J. Mol. Biol. 226:735-745.

24.Gerdes, K. 1988. The parB (hok/sok) locus of plasmid R1: a general purpose plasmid stabilization system. BioTechnology 6:1402-1405.

25.Kristoffersen, P., G.B. Jensen, K. Gerdes, and J. Piskur. 2000. Bacterial toxin-antitioxin gene system as containment control in yeast cells. Appl. Environ. Microbiol. 66:5524-5526.

26. Yamamoto, T., K. Gerdes, and A. Tunnaclifffe. 2002. Bacterial toxin RelE induces apoptosis in human cells. FEBS Lett. 519:191-194.

27.de la Cueva-Mendez, G., A.D. Mills, L. Clay-Farrace, R. Diaz-Orejas, and R.A. Laskey. 2003. Regulatable killing of eukaryotic cells by the prokaryotic proteins Kid and Kis. EMBO J. 22:246-251.

28.Corchero, J.L., R. Cubarsi, P. Vila, A. Aris, and A. Villaverde. 2001. Cell lysis in Escherichia coli cultures stimulates growth and biosynthesis of recombinant proteins in surviving cells. Microbiol. Res. 156:13-18

29.saiSree, L., M. Reddy, and J. Gowrishankar. 2001. IS186 insertion at a hot spot in the lon promoter as a basis for lon protease deficiency of Escherichia coli B: identification of a consensus target sequence for IS186 transposition. J. Bacteriol. 183:6943-6946.

30.Bernard, P. 1996. Positive selection of recombinant DNA by CcdB. BioTechniques 21:320-323.

Received 2 August 2004; accepted 24 December 2004.

Address correspondence to:

Cédric Y. Szpirer

Laboratory of Evolutionary Genetics

Institute of Molecular Biology and Medicine

Université Libre de Bruxelles

rue Jeener \& Brachet 12

6041 Gosselies, Belgium

e-mail: cedric.szpirer@ulb.ac.be 